\title{
A Bounded Jump for the Bounded Turing Degrees
}

\author{
Bernard Anderson and Barbara Csima
}

\begin{abstract}
We define the bounded jump of $A$ by $A^{b}=\{x \in \omega \mid \exists i \leq$ $\left.x\left[\varphi_{i}(x) \downarrow \wedge \Phi_{x}^{A \Uparrow \varphi_{i}(x)}(x) \downarrow\right]\right\}$ and let $A^{n b}$ denote the $n$th bounded jump. We demonstrate several properties of the bounded jump, including the fact that it is strictly increasing and order-preserving on the bounded Turing $(b T)$ degrees (also known as the weak truth-table degrees). We show that the bounded jump is related to the Ershov hierarchy. Indeed, for $n \geq 2$ we have $X \leq_{b T} \emptyset^{n b} \Longleftrightarrow X$ is $\omega^{n}$-c.e. $\Longleftrightarrow X \leq_{1} \emptyset^{n b}$, extending the classical result that $X \leq_{b T} \emptyset^{\prime} \Longleftrightarrow X$ is $\omega$-c.e. Finally, we prove that the analogue of Shoenfield inversion holds for the bounded jump on the bounded Turing degrees. That is, for every $X$ such that $\emptyset^{b} \leq_{b T} X \leq_{b T} \emptyset^{2 b}$, there is a $Y \leq_{b T} \emptyset^{b}$ such that $Y^{b} \equiv_{b T} X$.
\end{abstract}

\section{Introduction}

In computability theory, we are interested in comparing the relative computational complexities of infinite sets of natural numbers. There are many ways of doing this, and which method is used often depends on the purpose of the study, or how fine a comparison is desired. Two sets of the same computational complexity ( $X \leq Y$ and $Y \leq X)$ are said to be in the same degree. The computable sets form the lowest degree for all of the reducibilities we consider here.

Some of the most natural reducibilities are $m$-reducibility and 1-reducibility. Recall that a set $A$ is $m$-reducible (1-reducible) to a set $B$ if there is a computable (injective) function $f$ such that for all $x, x \in A$ iff $f(x) \in B$. The major failing of these reducibilities is that a set need not be reducible to its complement.

The most commonly studied reducibility is that of Turing reducibility, where $A$ is Turing reducible to $B$ if there is a program that, with reference to an infinite oracle tape containing $B$, computes $A$. Though each computation of a Turing reduction

Received December 14, 2011; accepted October 2, 2012

2010 Mathematics Subject Classification: Primary 03D30

Keywords: bounded jump, jump, bounded Turing degrees, $b T$-degrees, wtt degrees

(C) 2014 by University of Notre Dame $\quad 10.1215 / 00294527-2420660$ 
views only finitely much of the oracle tape, there is no computable bound on how much of the tape can be viewed in a computation.

Many natural Turing reductions have the property that the use of the oracle is bounded by a computable function. We will refer to such reductions as bounded Turing reductions and write $A \leq_{b T} B$. This is also commonly known in the literature as weak truth-table $(w t t)$ reducibility.

A truth-table reduction is a pair of computable functions $f$ and $g$, such that, for each $x, f(x)$ supplies a finite list $x_{1}, \ldots, x_{n}$ of positions of the oracle, and $g(x)$ provides a truth table on $n$ variables (a map $2^{n} \rightarrow 2$ ). A set $A$ is said to be truth-table reducible to $B$ if there is a truth-table reduction $f, g$ such that, for every $x, x \in A$ iff the row of the truth table $g$ obtained by viewing $B$ on the positions $x_{1}, \ldots, x_{n}$ has value 1 . It is easy to see that $A \leq_{t t} B$ iff $A$ is Turing reducible to $B$ via a functional that is total on all oracles. Note that if a functional is total on all oracles, then there is a computable bound on the use for each input. Bounded Turing reducibility is weaker than $t t$-reducibility, and this is where the name "weak truth-table reducibility" originated. However, since the weakening has nothing to do with the truth table, we follow the notation of $b T$, as used in Soare [16], [15].

The halting set is the first natural example of a noncomputable set. The Turing jump operator works by relativizing the halting set to other oracles. Basic properties of the Turing jump include that it is strictly increasing with respect to Turing reducibility and that it maps a single Turing degree into a single 1-degree. This latter property shows that the Turing jump is a well-defined operator on all of the degree structures we have mentioned so far.

The strictly increasing property of the Turing jump implies that the Turing jump of any set must compute the halting set. There are a variety of "jump inversion" results that show that the range of the Turing jump is maximal (with respect to a restricted domain). Friedberg jump inversion states that for every $X \geq_{T} \emptyset^{\prime}$ there exists $A$ with $A^{\prime} \equiv_{T} X \equiv_{T} A \oplus \emptyset^{\prime}$. Shoenfield [14] demonstrated that for every $\Sigma_{2}$-set $X \geq_{T} \emptyset^{\prime}$ there is a set $Y \leq_{T} \emptyset^{\prime}$ such that $Y^{\prime} \equiv_{T} X$.

What about jump inversion for strong reducibilities? Mohrherr [9] showed that for any $X \geq_{t t} \emptyset^{\prime}$, there exists $A$ with $A^{\prime} \equiv X$. Anderson [1] showed that the full analogue of Friedberg jump inversion holds: for every $X \geq_{t t} \emptyset^{\prime}$, there exists $A$ with $A^{\prime} \equiv_{t t} X \equiv_{t t} A \oplus \emptyset^{\prime}$. Both Mohrherr's and Anderson's proofs work with $b T$ in place of $t t$. However, Csima, Downey, and $\mathrm{Ng}$ [5] have proved that the analogue of Shoenfield jump inversion fails to hold for the $t t$ and bounded Turing degrees. Indeed, they showed that there is a $\Sigma_{2}$-set $C>_{t t} \emptyset^{\prime}$ such that for every $D \leq_{T} \emptyset^{\prime}$ we have $D^{\prime} \not_{b T} C$. The proof exploits the fact that the Turing jump is defined with respect to Turing (and not bounded Turing) reducibilities.

Our goal for this paper was to develop a jump operator for the bounded Turing degrees. We wanted this jump to be bounded in its use of the oracle and to hold all of the properties usually associated with a jump operator (in particular, strictly increasing and order-preserving). In this paper, we will define such a jump, examine its properties, and show that it is distinct from already used operators. We will prove that Shoenfield inversion holds for the bounded Turing degrees with this jump.

The sets that are computable from the $n$th Turing jump of $\emptyset$ have a very nice characterization - they are exactly the $\Delta_{n+1}^{0}$-sets. The $n$th bounded jumps of $\varnothing$ also 
have a natural characterization. In this case, the connection is with the Ershov hierarchy. For $n \geq 2$, the sets that can be $b T$-computed from (indeed, are $t t$ or 1-below) the $n$th iterate of the bounded jump are exactly the $\omega^{n}$-c.e. sets.

There have been other jumps for strong reducibilities introduced in the past, and we discuss some of these in Section 7. It has recently come to our attention that Coles, Downey, and Laforte [3] had studied an operator similar to our bounded jump (defined as $A^{b_{1}}$ in this paper), but unfortunately no written record of their work exists beyond a proof that their jump is strictly increasing.

\section{Notation}

We mainly follow the standard notation for computability theory as found in Cooper [4] and Soare [16], [15]. We let $\varphi_{0}, \varphi_{1}, \varphi_{2}, \ldots$ be an effective enumeration of the partial computable functions and let $\Phi_{0}, \Phi_{1}, \Phi_{2}, \ldots$ be an effective enumeration of the Turing functionals. We assume that our enumerations are acceptable.

We let $\emptyset^{\prime}=\left\{x \mid \varphi_{x}(x) \downarrow\right\}$; and for an arbitrary set $A$, let $A^{\prime}=\left\{x \mid \Phi_{x}^{A}(x) \downarrow\right\}$. In the case when the enumeration $\left\{\varphi_{n}\right\}_{n \in \omega}$ is such that $\varphi_{n}=\Phi_{n}^{\emptyset}$, then there is no confusion with the two definitions of $\emptyset^{\prime}$. But under any enumeration, the two definitions are 1-equivalent.

For a set $A$, we let $A \Uparrow x=\{n \in A \mid n \leq x\}$. We follow an expression with a stage number in brackets (i.e., $[s]$ ) to indicate that the stage number applies to everything in the expression that is indexed by stage.

For sets $A$ and $B$ we write that $A \leq_{b T} B$ and say that $A$ is bounded Turing reducible to $B$, if there exist $i$ and $j$ such that $\varphi_{j}$ is total and for all $x$, $A(x)=\Phi_{i}^{B \Uparrow \varphi_{j}(x)}(x) \downarrow$. This agrees with the informal definition of $b T$ given in the introduction.

\section{The Bounded Jump}

Definition 1 Given a set $A$, we define the bounded jump

$$
A^{b}=\left\{x \in \omega \mid \exists i \leq x\left[\varphi_{i}(x) \downarrow \wedge \Phi_{x}^{A \Uparrow \varphi_{i}(x)}(x) \downarrow\right]\right\} .
$$

We let $A^{n b}$ denote the $n$th bounded jump.

Remark 3.1 We have $\emptyset^{b} \equiv{ }_{1} \emptyset^{\prime}$.

This holds since bounding the use of an empty oracle has no effect. We will use $\emptyset^{b}$ and $\emptyset^{\prime}$ interchangeably from now on.

We consider a more general definition of the bounded jump.

Definition 2 We have $A^{b_{0}}=\left\{\langle e, i, j\rangle \in \omega \mid \varphi_{i}(j) \downarrow \wedge \Phi_{e}^{A \Uparrow \varphi_{i}(j)}(j) \downarrow\right\}$.

We show that, up to truth-table equivalence, $A^{b}$ and $A^{b_{0}}$ are the same. We will at times identify one with the other.

Remark 3.2 For any set $A$ we have $A^{b} \leq_{t t} A^{b_{0}}$.

This is true since $x \in A^{b} \Longleftrightarrow \exists i \leq x\left[\langle x, i, x\rangle \in A^{b_{0}}\right]$.

Theorem 3.3 For any set $A$ we have $A^{b_{0}} \leq_{1} A^{b}$. 
Proof We define a computable and injective function $k$ by $\varphi_{k(i, j)}(x)=\varphi_{i}(j)$. We then define the function $g$ (also computable and injective) by

$$
\Phi_{g(\langle e, i, j\rangle)}^{C}(x)= \begin{cases}\Phi_{e}^{C \Uparrow \varphi_{k(i, j)}(x)}(j) & \varphi_{i}(j) \downarrow, \\ \uparrow & \text { else. }\end{cases}
$$

By the padding lemma we may assume without loss of generality that for all $e, i$, and $j$ we have $g(\langle e, i, j\rangle) \geq k(i, j)$.

We now show that $\langle e, i, j\rangle \in A^{b_{0}} \Longleftrightarrow g(\langle e, i, j\rangle) \in A^{b}$.

For the forward direction, we use $k(i, j)$ as the witness that $g(\langle e, i, j\rangle) \in A^{b}$. We have the following:

$$
\begin{aligned}
& \langle e, i, j\rangle \in A^{b_{0}} \\
& \Rightarrow \quad \varphi_{i}(j) \downarrow \text { and } \Phi_{e}^{A \Uparrow \varphi_{i}(j)}(j) \downarrow \\
& \Rightarrow \quad \text { for any } x, \varphi_{k(i, j)}(x) \downarrow \text { and } \Phi_{g(\langle e, i, j\rangle)}^{A \Uparrow \varphi_{k(i, j)}(x)}(x) \downarrow \text { [by definitions of } g \text { and } k \text { ] } \\
& \Rightarrow \quad \varphi_{k(i, j)}(g(\langle e, i, j\rangle)) \downarrow \\
& \quad \text { and } \Phi_{g(\langle e, i, j\rangle)}^{A \Uparrow \varphi_{k(i, j)}(g(\langle e, i, j\rangle))}(g(\langle e, i, j\rangle)) \downarrow[\operatorname{let} x=g(\langle e, i, j\rangle)] \\
& \Rightarrow \quad \exists l \leq g(\langle e, i, j\rangle)\left[\varphi_{l}(g(\langle e, i, j\rangle)) \downarrow \text { and } \Phi_{g(\langle e, i, j\rangle)}^{A \Uparrow \varphi_{l}(g(\langle e, i, j\rangle))}(g(\langle e, i, j\rangle)) \downarrow\right] \\
& \quad \quad[\operatorname{let} l=k(i, j)] \\
& \Rightarrow \quad g(\langle e, i, j\rangle) \in A^{b} .
\end{aligned}
$$

For the backward direction, we ignore the witness $l$ that $g(\langle e, i, j\rangle) \in A^{b}$ and rely on the definition of $g$ :

$$
\begin{aligned}
g(\langle e, i, j\rangle) & \in A^{b} \\
\Rightarrow \quad & \exists l \leq g(\langle e, i, j\rangle)\left[\varphi_{l}(g(\langle e, i, j\rangle)) \downarrow \text { and } \Phi_{g(\langle e, i, j\rangle)}^{A \Uparrow \varphi_{l}(g(\langle e, i, j\rangle))}(g(\langle e, i, j\rangle)) \downarrow\right] \\
\Rightarrow \quad & \exists l \leq g(\langle e, i, j\rangle)\left[\varphi_{l}(g(\langle e, i, j\rangle)) \downarrow \text { and } \varphi_{i}(j) \downarrow\right. \\
& \left.\quad \text { and } \Phi_{e}^{\left(A \Uparrow \varphi_{l}(g(\langle e, i, j\rangle))\right) \Uparrow \varphi_{k(i, j)}(g(\langle e, i, j\rangle))}(j) \downarrow\right][\text { by definition of } g] \\
\Rightarrow \quad & \exists l \leq g(\langle e, i, j\rangle)\left[\varphi_{l}(g(\langle e, i, j\rangle)) \downarrow \text { and } \varphi_{i}(j) \downarrow\right. \\
& \left.\quad \text { and } \Phi_{e}^{A \Uparrow \min \left(\varphi_{l}(g(\langle e, i, j\rangle)), \varphi_{i}(j)\right)}(j) \downarrow\right] \\
\Rightarrow \quad \varphi_{i}(j) \downarrow \text { and } \Phi_{e}^{A \Uparrow \varphi_{i}(j)}(j) \downarrow & \quad\langle e, i, j\rangle \in A^{b_{0}} .
\end{aligned}
$$

We see later in Remark 5.7 that we cannot strengthen this to $A^{b} \equiv_{1} A^{b_{0}}$.

Another possibility is a more "diagonal" definition for the bounded jump.

Definition 3 We have $A^{b_{1}}=\left\{x \mid \varphi_{x}(x) \downarrow \wedge \Phi_{x}^{A \Uparrow \varphi_{x}(x)}(x) \downarrow\right\}$.

We view this definition as less desirable, since it depends heavily on the particular enumeration $\left\{\varphi_{x}\right\}_{x \in \omega}$ of the partial computable functions. Indeed, depending on the enumeration, one could have $A^{b_{1}}=\emptyset^{\prime}$ for all sets $A$, or with a different enumeration, $A^{b_{1}} \equiv_{1} A^{b_{0}}$.

Finally, we might also consider a simpler bounded jump.

Definition 4 We have $A^{i}=\left\{x \in \omega \mid \Phi_{x}^{A \Uparrow x}(x) \downarrow\right\}$.

However, this definition seems unsatisfactory since it is not strictly increasing. 
Remark 3.4 Let $A$ be a set with $A \geq_{b T} \emptyset^{\prime}$. Then $A \geq_{b T} A^{i}$.

Proof We show that $A^{i} \leq_{b T} A \oplus \emptyset^{\prime}$ for any $A$. Let $f(n)$ denote the maximum over all strings $\sigma$ of length $n$, of the location of $\emptyset^{\prime}$ needed to determine if $\Phi_{n}^{\sigma}(n) \downarrow$. Then $\emptyset^{\prime} \| f(n)$ and $A \Uparrow n$ suffice to compute $A^{i}(n)$.

\section{Properties}

We summarize some facts about the bounded jump. Let $A$ be any set:

1. $\emptyset^{b} \equiv{ }_{1} \emptyset^{\prime}$.

2. $A \leq_{1} A^{b}$.

3. $A^{b} \leq_{1} A^{\prime}$ (since $A^{b}$ is c.e. in $A$ ).

4. $\emptyset^{\prime} \leq_{t t} A^{b}$ (as a consequence of Corollary 4.4 below).

5. $A^{b} \equiv_{T} A \oplus \emptyset^{\prime}$ (by Proposition 4.1 below).

6. If $A \geq_{T} \emptyset^{\prime}$, then $A^{b} \leq_{T} A$.

7. Let $A$ be such that $A^{\prime} \bigsqcup_{T} A \oplus \emptyset^{\prime}$ (e.g., any $A \geq_{T} \emptyset^{\prime}$ ); then $A^{\prime} \bigsqcup_{T} A^{b}$ (so $\left.A^{\prime} \varliminf_{b T} A^{b}\right)$.

8. $A^{b} \varliminf_{b T} A$ (by Theorem 4.2 below).

9. If $A \geq_{b T} \emptyset^{\prime}$, then $A^{b} \varliminf_{b T} A \oplus \emptyset^{\prime}$.

The effect of the bounded jump on the Turing degrees is easy to characterize.

Proposition 4.1 Let $A$ be any set. Then $A^{b} \leq_{T} A \oplus \emptyset^{\prime}$.

Proof We wish to determine if a given $n$ is such that $\exists i \leq n\left[\varphi_{i}(n) \downarrow \wedge\right.$ $\left.\Phi_{n}^{A \Uparrow \varphi_{i}(n)}(n) \downarrow\right]$. We note that the existential quantifier is bounded. Given $i \leq n$, we use $\emptyset^{\prime}$ to determine if $\varphi_{i}(n) \downarrow$. If it does, we then get $\sigma=A \Uparrow \varphi_{i}(n)$ from $A$ and use $\emptyset^{\prime}$ to determine if $\Phi_{n}^{\sigma}(n) \downarrow$. This does not require $A^{\prime}$ since the use of $A$ is bounded. We can then determine if $n \in A^{b}$.

While the bounded jump is not very interesting from the perspective of the Turing degrees, we hope to show that it follows our intuition for a jump on the bounded Turing degrees.

We start by showing that the bounded jump is strictly increasing. The proof is a diagonalization argument using the recursion theorem.

Theorem 4.2 Let $A$ be any set. Then $A^{b} \bigsqcup_{b T} A$.

Proof Suppose not. Let $\Gamma$ and $g$ witness $A^{b} \leq_{b T} A$. We define a computable function $f$ by

$$
\Phi_{f(e)}^{C}(x)= \begin{cases}0, & x \neq e \text { or }\left(x=e \text { and } \Gamma^{C}(e)=0\right), \\ \Phi_{e}^{C}(e)+1, & x=e \text { and } \Gamma^{C}(e)=1, \\ \uparrow & x=e \text { and } \Gamma^{C}(e) \uparrow .\end{cases}
$$

By the recursion theorem, let $M$ be an infinite computable set such that for all $m \in M$ we have $\Phi_{m}^{C}=\Phi_{f(m)}^{C}$. Let $k$ be such that $g=\varphi_{k}$, and pick $m \in M$ such that $m>k$. We note that $\Gamma^{A}$ is total, so $\Phi_{f(m)}^{A}$ is total and thus $\Phi_{m}^{A}$ is total. tion.

Suppose $\Gamma^{A}(m)=1$. Then $\Phi_{m}^{A}(m)=\Phi_{f(m)}^{A}(m)=\Phi_{m}^{A}(m)+1$ for a contradic-

Hence $\Gamma^{A}(m)=0$. Thus $m \notin A^{b}$. So for all $i \leq m$ with $\varphi_{i}(m) \downarrow$ we have $\Phi_{m}^{A \Uparrow \varphi_{i}(m)}(m) \uparrow$. In particular, since $k<m$ and $\varphi_{k}(m)=g(m) \downarrow$ we have 
$\Phi_{m}^{A \Uparrow g(m)}(m) \uparrow$. Thus $\Phi_{f(m)}^{A \Uparrow g(m)}(m) \uparrow$ so $\Gamma^{A \Uparrow g(m)}(m) \uparrow$. This contradicts our choice of $\Gamma$ and $g$.

We conclude that $A^{b} \underline{\perp}_{b T} A$.

We next show the bounded jump is order-preserving on the bounded Turing degrees. The proof is a careful application of the $s-m-n$ theorem.

Theorem 4.3 Let $A$ and $B$ be sets with $A \leq_{b T} B$. Then $A^{b_{0}} \leq_{1} B^{b_{0}}$.

Proof Let $\Psi$ and $f$ witness $A \leq_{b T} B$. By the $s-m-n$ Theorem, let $h$ be a strictly increasing computable function such that $\varphi_{h(i)}(x)=f\left(\varphi_{i}(x)\right)$. Since $f$ is total, $\varphi_{h(i)}(x) \downarrow \Longleftrightarrow \varphi_{i}(x) \downarrow$.

We define a computable, injective function $g$ by

$$
\Phi_{g(\langle e, k, j\rangle)}^{C}(x)= \begin{cases}\Phi_{e}^{\left(\Psi^{C \Uparrow \varphi_{h(i)}(j)}\right) \Uparrow \varphi_{i}(j)}(j), & k=h(i) \text { for some } i \text { and } \varphi_{i}(j) \downarrow \\ \uparrow & \text { else. }\end{cases}
$$

We now note that

$$
\begin{aligned}
\langle e, i, j\rangle \in A^{b_{0}} & \Longleftrightarrow \varphi_{i}(j) \downarrow \text { and } \Phi_{e}^{A \Uparrow \varphi_{i}(j)}(j) \downarrow, \\
& \Longleftrightarrow \varphi_{h(i)}(j) \downarrow \text { and } \Phi_{e}^{A \Uparrow \varphi_{i}(j)}(j) \downarrow, \\
& \Longleftrightarrow \varphi_{h(i)}(j) \downarrow \text { and } \Phi_{e}^{\left(\Psi^{B \Uparrow \varphi_{h(i)}(j)}\right) \Uparrow \varphi_{i}(j)}(j) \downarrow, \\
& \Longleftrightarrow \varphi_{h(i)}(j) \downarrow \text { and } \Phi_{g(\langle e, h(i), j\rangle)}^{B \| \varphi_{h(i)}(j)}(j) \downarrow, \\
& \Longleftrightarrow\langle g(\langle e, h(i), j\rangle), h(i), j\rangle \in B^{b_{0}} .
\end{aligned}
$$

Therefore $A^{b_{0}} \leq_{1} B^{b_{0}}$.

Since for any set $X$ we have $X^{b_{0}} \equiv_{t t} X^{b}$, we immediately obtain the following corollary.

Corollary 4.4 Let $A$ and $B$ be sets with $A \leq_{b T} B$. Then $A^{b} \leq_{t t} B^{b}$.

We would also like to show that $A^{b}$ is not equivalent to $A \oplus \emptyset^{\prime}$ for the bounded Turing degrees. We noted earlier that this holds on the cone above $\emptyset^{\prime}$. We can also demonstrate that this holds elsewhere. We recall two notions of sets being "ordinary" (see Nies [10] for more information on randomness).

Definition $5 \quad X$ is $n$-generic if for every $\Sigma_{n}$-set $S \subseteq 2^{\omega}$ either $X$ meets $S$ (i.e., there is an initial segment of $X$ in $S$ ) or there is an $l \in \omega$ such that every string $\sigma$ extending $X \Uparrow l$ is such that $\sigma \notin S$.

Definition $6 \quad X$ is $n$-random if for every uniformly $\Sigma_{n}$-family of sets $\left\langle U_{i} \subseteq 2^{\omega}\right|$ $i \in \omega\rangle$ such that $\mu\left(U_{i}\right) \leq 2^{-i}$ for all $i$, there exists an $l$ such that $X$ does not meet $U_{l}$.

We show that if $A$ is 3-generic or 4-random, then $A^{b} \not_{b T} A \oplus \emptyset^{\prime}$. In the proof, we will assume toward a contradiction that $\Psi$ and $f$ witness $A^{b_{0}} \leq_{b T} A \oplus \emptyset^{\prime}$. We will then use the recursion theorem to find a computable set whose elements $n$ are such that $f(n)+1 \in A \Longleftrightarrow n \in A^{b_{0}}$. Since $A \Uparrow f(n)$ computes $A^{b_{0}}(n)$, we have $A(f(n)+1)$ predicted by $A \| f(n)$. This regularity property can then be used to show that $A$ is not 3-generic or 4-random, for a contradiction.

Theorem 4.5 Let $A$ be 3-generic. Then $A^{b} \bigsqcup_{b T} A \oplus \emptyset^{\prime}$. 
Proof Suppose not. Then $A^{b_{0}} \leq_{b T} A \oplus \emptyset^{\prime}$. Let $\Psi$ and $f$ be such that $\Psi^{A \Uparrow f(n) \oplus \emptyset^{\prime} \Uparrow f(n)}(n)=A^{b_{0}}(n)$ for all $n$.

For any $e$, let $\varphi_{g_{e}(i)}(j)=f(\langle e, i, j\rangle)+1$. By the recursion theorem, let $Z_{e}$ be an infinite computable set such that for all $m \in Z_{e}$ we have $\varphi_{g_{e}(m)}=\varphi_{m}$. We choose $g_{e}$ and $Z_{e}$ such that they are uniformly computable. Let $t$ be a computable function such that for all $e$ we have $t(e) \in Z_{e}$. Then for all $e, j \in \omega$, $\varphi_{t(e)}(j)=\varphi_{g_{e}(t(e))}(j)=f(\langle e, t(e), j\rangle)+1$. In particular, $\varphi_{t(e)}$ is total.

We define a computable function $h$ by

$$
\Phi_{h(e)}^{C}(j)= \begin{cases}1, & C\left(\varphi_{t(e)}(j)\right)=1, \\ \uparrow & \text { else. }\end{cases}
$$

By the recursion theorem, let $H$ be an infinite computable set such that for all $m \in H$ we have $\Phi_{h(m)}^{C}=\Phi_{m}^{C}$.

We then have for every $n \in H$ that

$$
\Phi_{n}^{C}(j)= \begin{cases}1, & C(f(\langle n, t(n), j\rangle+1))=1, \\ \uparrow & \text { else. }\end{cases}
$$

Hence for every $n \in H$ we have

$$
\begin{aligned}
\langle n, t(n), j\rangle \in A^{b_{0}} & \Longleftrightarrow \varphi_{t(n)}(j) \downarrow \wedge \Phi_{n}^{A \Uparrow \varphi_{t(n)}(j)}(j) \downarrow, \\
& \Longleftrightarrow \Phi_{n}^{A \Uparrow f(\langle n, t(n), j\rangle)+1}(j) \downarrow, \\
& \Longleftrightarrow A(f(\langle n, t(n), j\rangle)+1)=1 .
\end{aligned}
$$

Thus for $n \in H$ we have

$$
\begin{gathered}
\Psi^{A \Uparrow f(\langle n, t(n), j\rangle) \oplus \emptyset^{\prime} \Uparrow f(\langle n, t(n), j\rangle)}(\langle n, t(n), j\rangle)=1 \\
\Longleftrightarrow A(f(\langle n, t(n), j\rangle)+1)=1 .
\end{gathered}
$$

We define a set $S$ by

$$
\begin{aligned}
S= & \left\{\sigma \in 2^{\omega} \mid \exists j \exists n \in H[\text { length }(\sigma)>f(\langle n, t(n), j\rangle) \text { and }\right. \\
& \Psi^{\sigma \Uparrow f(\langle n, t(n), j\rangle) \oplus \emptyset^{\prime} \Uparrow f(\langle n, t(n), j\rangle)}(\langle n, t(n), j\rangle) \downarrow \neq \sigma(f(\langle n, t(n), j\rangle)+1) \\
& \text { or diverges }]\} .
\end{aligned}
$$

We note that $S$ is $\Sigma_{2}\left(\emptyset^{\prime}\right)$, and so $S$ is $\Sigma_{3}$. Since $A$ is 3 -generic and $A$ does not meet $S$, there is an $m$ such that for all $\tau$ extending $A \Uparrow m$ we have $\tau \notin S$. However, any string $\tau$ can be extended to one in $S$ by picking a value for $\tau(f(\langle n, t(n), j\rangle)+1)$ that disagrees with the prediction of $\Psi$ (if it converges) for some sufficiently large $n \in H$ and $j$. This is a contradiction, so we conclude that $A^{b} \not_{b T} A \oplus \emptyset^{\prime}$.

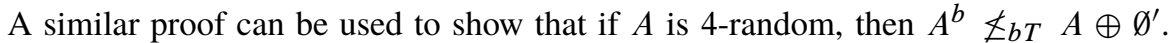
Hence, for the bounded Turing degrees, the class of sets where $A^{b}$ is equivalent to $A \oplus \emptyset^{\prime}$ has measure zero.

Corollary 4.6 Let $A$ be 4-random. Then $A^{b} \bigsqcup_{b T} A \oplus \emptyset^{\prime}$.

Proof Suppose not. Then $A^{b_{0}} \leq_{b T} A \oplus \emptyset^{\prime}$. Let $\Psi$ and $f$ be such that $\Psi^{A \Uparrow f(n) \oplus \emptyset^{\prime} \Uparrow f(n)}(n)=A^{b_{0}}(n)$ for all $n$.

Let $t, H$, and $S$ be as in the proof of Theorem 4.5. Since $f, t$, and $H$ are computable, we can find a computable, strictly increasing function $l$ such that for all $m \in \omega$ we have $l(m)=f(\langle n, t(n), j\rangle)$ for some $j$ and some $n \in H$. 
For each $i \in \omega$ let $U_{i}=\{\sigma \mid \sigma \notin S$ and length $(\sigma)=l(i)+1\}$. We note that the $U_{i}$ are uniformly $\Pi_{3}$ since $S$ is $\Sigma_{3}$ and $l$ is computable. Since $A$ does not meet $S$, we know that $A$ meets every $U_{i}$.

We note from the definition of $S$ that if $\tau$ is any string of length $l(m)$ for some $m$, then at least one of $\tau^{\wedge} 0$ and $\tau^{\wedge} 1$ is in $S$. We also note $S$ is closed under extensions, so if $\sigma \notin U_{i}$, length $(\sigma) \geq l(i)$, and $\rho$ extends $\sigma$, then $\rho \notin U_{j}$ for any $j \geq i$. Hence $\mu\left(U_{i}\right) \leq 2^{-i}$. We conclude that $A$ is not 4-random, for a contradiction. Thus $A^{b} \nless_{b T} A \oplus \emptyset^{\prime}$.

\section{Ershov Hierarchy}

The iterates of the jump correspond to completeness in the arithmetic hierarchy; the $n$th jump is $\Sigma_{n}$-complete. We will show that the iterates of the bounded jump correspond to completeness in the Ershov hierarchy.

Fix a canonical, computable coding of the ordinals less than $\omega^{\omega}$. Since we do not use ordinals above $\omega^{\omega}$ in this paper, the details of the coding are not significant. We say that a function on an ordinal $\alpha$ is (partial) computable if the corresponding function on codes for the ordinal $\alpha$ is (partial) computable.

For $\alpha \geq \omega$, we say that a set $A$ is $\alpha$-c.e. if there is a partial computable $\psi: \omega \times \alpha \rightarrow\{0,1\}$ such that for every $n \in \omega$, there exists a $\beta<\alpha$ where $\psi(n, \beta) \downarrow$ and $A(n)=\psi(n, \gamma)$ where $\gamma$ is least such that $\psi(n, \gamma) \downarrow$ (see Jockusch and Shore [8]).

It is a well-known result that $X \leq_{b T} \emptyset^{\prime} \Longleftrightarrow X \leq_{t t} \emptyset^{\prime} \Longleftrightarrow X$ is $\omega$-c.e. (see [10]). Using the bounded jump, this is $X \leq_{b T} \emptyset^{b} \Longleftrightarrow X \leq_{t t} \emptyset^{b} \Longleftrightarrow X$ is $\omega$-c.e. We wish to extend this observation to higher powers of $\omega$. In fact, we are able to establish a slightly stronger result.

Theorem 5.1 For any set $X$ and $n \geq 2$ we have that $X \leq_{b T} \emptyset^{n b} \Longleftrightarrow X$ is $\omega^{n}$-c.e. $\Longleftrightarrow X \leq_{1} \emptyset^{n b}$.

A set $A$ is a $t t$-cylinder if for all $X$ we have $X \leq_{t t} A \Rightarrow X \leq_{1} A$ (see Odifreddi [11]).

\section{Corollary 5.2 For all $n \geq 2$ we have that $\emptyset^{n b}$ is a tt-cylinder.}

The theorem follows from the lemmas below. We first introduce some notation. Let $+_{c}$ denote commutative addition of ordinals (term-wise sum of coefficients of ordinals in Cantor normal form; see Ash and Knight [2]). We will use two properties of commutative addition. First, given $\alpha_{1} \cdots \alpha_{n}$ and $\beta_{1} \cdots \beta_{n}$ such that $\beta_{i} \leq \alpha_{i}$ for all $i \leq n$ and $\beta_{j}<\alpha_{j}$ for some $j \leq n$, then $\beta_{1}+{ }_{c} \beta_{2}+{ }_{c} \cdots+{ }_{c} \beta_{n}<\alpha_{1}+{ }_{c} \alpha_{2}+_{c} \cdots+{ }_{c} \alpha_{n}$. Also, if for some $\gamma$ we have $\alpha_{i}<\omega^{\gamma}$ for all $i$, then $\alpha_{1}+{ }_{c} \alpha_{2}+{ }_{c} \cdots+{ }_{c} \alpha_{n}<\omega^{\gamma}$.

We start by proving that being $\omega^{k}$-c.e. is closed downward in the bounded Turing degrees. For the proof, we suppose that $\Phi$ and $f$ witness $A \leq_{b T} B$ and $\psi$ witnesses that $B$ is $\omega^{k}$-c.e. We will then build $\chi$ to witness that $A$ is $\omega^{k}$-c.e. In order to estimate $A(n)$, we will estimate $B \Uparrow f(n)$ using $\psi\left(i, \alpha_{i}\right)$ for $i \leq f(n)$ and record the output of $\Phi$ on this estimate at $\chi\left(n, \alpha_{1}+{ }_{c} \cdots+{ }_{c} \alpha_{f(n)}\right)$.

Lemma 5.3 Let $k>0$, and let $A$ and $B$ be sets such that $A \leq_{b T} B$ and $B$ is $\omega^{k}$-c.e. Then $A$ is $\omega^{k}$-c.e. 
Proof Let $\Phi$ and $f$ witness $A \leq_{b T} B$, and let $\psi$ witness that $B$ is $\omega^{k}$-c.e. We will define a function $\chi$ to witness that $A$ is $\omega^{k}$-c.e. by stages as follows. Fix $n$. (We simultaneously follow the same procedure for each $n$.)

At each stage $s$, for $i \leq f(n)$, let $\alpha_{i}^{s}$ be the least ordinal such that $\psi_{s}\left(i, \alpha_{i}^{s}\right) \downarrow$, if it exists. Define a string $\sigma_{s}\left(\alpha_{0}^{s}, \ldots, \alpha_{f(n)}^{s}\right)$ of length $f(n)$ by letting $\sigma_{s}(i)=\psi_{s}\left(i, \alpha_{i}^{s}\right)$.

Let $s_{0}$ be the least stage where $\alpha_{i}^{s_{0}}$ are defined for all $i \leq f(n)$. Set

$$
\chi\left(n, \alpha_{0}^{s_{0}}+{ }_{c} \cdots+{ }_{c} \alpha_{f(n)}^{s_{0}}\right)=\Phi^{\sigma_{s_{0}}\left(\alpha_{0}^{s_{0}}, \ldots, \alpha_{f(n)}^{s_{0}}\right)}(n) .
$$

Note that $\alpha_{0}^{s_{0}}+{ }_{c} \cdots+{ }_{c} \alpha_{f(n)}^{s_{0}}<\omega^{k}$.

At stage $s+1>s_{0}$, if $\alpha_{i}^{s+1}<\alpha_{i}^{s}$ for some $i \leq f(n)$, then define

$$
\chi\left(n, \alpha_{0}^{s+1}+{ }_{c} \cdots+{ }_{c} \alpha_{f(n)}^{s+1}\right)=\Phi^{\sigma_{s+1}\left(\alpha_{0}^{s+1}, \ldots, \alpha_{f(n)}^{s+1}\right)}(n) .
$$

This is possible since $\alpha_{0}^{s+1}+{ }_{c} \cdots+{ }_{c} \alpha_{f(n)}^{s+1}<\alpha_{0}^{s}+{ }_{c} \cdots+{ }_{c} \alpha_{f(n)}^{s}$.

It is clear that $\chi$ is partial recursive. Let $n$ be arbitrary, and for $i \leq f(n)$ let $\beta_{i}$ be least such that $\psi\left(i, \beta_{i}\right) \downarrow$. Let $\gamma=\beta_{0}+{ }_{c} \cdots+{ }_{c} \beta_{f(n)}$. Then $\gamma$ is the least such that $\chi(n, \gamma) \downarrow$ and $\chi(n, \gamma)=A(n)$. Thus $\chi$ witnesses $A$ is $\omega^{k}$-c.e.

We next prove that if $A$ is $\omega^{k}$-c.e., then $A^{b}$ is $\omega^{k+1}$-c.e. Combined with the previous lemma this will give us that $X \leq_{b T} \emptyset^{n b} \Rightarrow X$ is $\omega^{n}$-c.e.

For the proof, we will let $\psi$ witness that $A$ is $\omega^{k}$-c.e. and will define $\chi$ to witness that $A^{b}$ is $\omega^{k+1}$-c.e. We will start with $\chi\left(n, \omega^{k} \cdot n\right)=0$, and each time we witness a new, longer $\varphi_{i}(n) \downarrow$ for some $i \leq n$ we will move down to a new $\omega^{k}$-level. At a fixed level, we will record estimates of $A^{b}$ based on estimates of $A \Uparrow \varphi_{i}(n)$ in a manner similar to Lemma 5.3.

Lemma 5.4 Let $k>0$, and let $A$ be a set such that $A$ is $\omega^{k}$-c.e. Then $A^{b}$ is $\omega^{k+1}$-c.e.

Proof Let $\psi$ witness that $A$ is $\omega^{k}$-c.e. We will define a function $\chi$ to witness that $A^{b}$ is $\omega^{k+1}$-c.e. Fix $n$. (We simultaneously follow the same procedure for each $n$.)

For an ordinal $\beta$, let $u(\beta)$ be the coefficient of the units digit of $\beta$ in Cantor normal form. We again let $\alpha_{i}^{s}$ be the least ordinal such that $\psi_{s}\left(i, \alpha_{i}^{s}\right) \downarrow$, if it exists, and define a string $\sigma_{s}\left(\alpha_{0}^{s}, \ldots, \alpha_{m}^{s}\right)$ of length $m$ by letting $\sigma_{s}(i)=\psi_{s}\left(i, \alpha_{i}^{s}\right)$. Indeed, we will assume without loss of generality that $\alpha_{i}^{s}$ is defined at each stage $s$ by running the computation $\psi$ for longer than $s$ steps if necessary.

Let $r\left(l, \alpha_{0}^{s}, \ldots, \alpha_{m}^{s}\right)=\omega^{k} \cdot l+{ }_{c} \alpha_{0}^{s}+{ }_{c} \cdots+{ }_{c} \alpha_{m}^{s}+{ }_{c} u\left(\alpha_{0}^{s}+{ }_{c} \cdots+{ }_{c} \alpha_{m}^{s}\right)$. Note that if all $\alpha_{i}^{s}<\omega^{k}$, then $r\left(l, \alpha_{0}, \ldots, \alpha_{m}\right)<\omega^{k+1}$. Note also that if $\beta_{i} \leq \alpha_{i}$ for all $i \leq m$ and $l^{\prime} \leq l$, and if one of the inequalities is strict, then $r\left(l^{\prime}, \beta_{0}, \ldots, \beta_{m}\right)+2<r\left(l, \alpha_{0}, \ldots, \alpha_{m}\right)+1$.

We let $\chi\left(n, \omega^{k} \cdot n\right)=0$ and set bookkeeping variables $l_{0}=n$ and $m_{0}=0$. Every time we see $\varphi_{i}(n) \downarrow>m$ for some $i \leq n$ we will decrease $l$ by one and let $m=\varphi_{i}(n)$. We note this can happen at most $n$ many times.

At stage $s+1$, if $\varphi_{i, s+1}(n) \downarrow>m_{s}$ for some $i \leq n$, define $l_{s+1}=l_{s}-1$, and let $m_{s+1}=\varphi_{i}(n)$. Otherwise, let $l_{s+1}=l_{s}$ and $m_{s+1}=m_{s}$.

If $l_{s+1}<l_{s}$ or if $\alpha_{i}^{s+1}<\alpha_{i}^{s}$ for some $i \leq m_{s+1}$, then let $\chi\left(n, r\left(l, \alpha_{0}, \ldots, \alpha_{m}\right)+\right.$ 2) $[s+1]=0$.

If $\Phi_{n}^{\sigma\left(l, \alpha_{0}, \ldots, \alpha_{m}\right)}(n) \downarrow[s+1]$, then set $\chi\left(n, r\left(l, \alpha_{0}, \ldots, \alpha_{m}\right)+1\right)[s+1]=1$.

This completes the construction. 
We note that $\chi$ is partial recursive. Let $n$ be arbitrary, and let $m$ be the largest value of $\varphi_{i}(n)$ for $i \leq n$ such that $\varphi_{i}(n) \downarrow$. For $j \leq m$, let $\beta_{j}$ be the least such that $\psi\left(j, \beta_{j}\right) \downarrow$. Let $l$ be the least such that for some $\delta<\omega^{k}$ we have $\chi\left(n, \omega^{k} \cdot l+\delta\right) \downarrow$. Then $\chi\left(n, r\left(l, \beta_{0} \cdots \beta_{m}\right)+2\right)=0$ and $\chi\left(n, r\left(l, \beta_{0} \cdots \beta_{m}\right)+1\right) \downarrow=1$ iff $n \in A^{b}$. For all $\gamma \leq r\left(l, \beta_{0} \cdots \beta_{m}\right)$ we have $\chi(n, \gamma) \uparrow$. Therefore $\chi$ witnesses that $A^{b}$ is $\omega^{k+1}$-c.e.

We note that the proofs for the above lemmas hold for any ordinal $\omega^{\gamma}$ such that $0<\omega^{\gamma}<\omega_{1}^{C K}$.

To complete the proof of the theorem, we wish to show that if $A$ is $\omega^{k}$-c.e., then $A \leq{ }_{1} \emptyset^{k b}$. We start by proving the statement for $k=2$.

For the proof, suppose that $\psi$ witnesses that $A$ is $\omega^{2}$-c.e. Let $n \in \omega$, and let $m$ be the first such that we see $\psi(n, \omega \cdot m+j) \downarrow$ for some $j$. To determine if $n \in A$, we need to know enough of $\emptyset^{b}$ to answer the $\Sigma_{1}$-questions $\exists j[\psi(n, \omega \cdot i+j) \downarrow]$ for each $i<m$. In each case, if the answer is yes, first witnessed by $\omega \cdot i+k$, we then need to know if $\psi(n, \omega \cdot i+j) \downarrow$ for all $j<k$. There is no computable bound which can be determined in advance stating how much of $\emptyset^{b}$ is needed to answer all of these questions. However, we can in advance bound the indices of the computable functions needed to determine how much of $\emptyset^{b}$ will be used. Hence we can bound the amount of $\emptyset^{2 b}$ required to have enough access to $\emptyset^{b}$ to answer these questions.

Lemma 5.5 Let $A$ be a set such that $A$ is $\omega^{2}$-c.e. Then $A \leq_{1} \emptyset^{2 b}$.

Proof Let $\psi$ witness that $A$ is $\omega^{2}$-c.e. We will define several functions, ending in a computable $f$ such that $n \in A \Longleftrightarrow f(n) \in \emptyset^{2 b}$.

Let $g$ be a computable function such that $g(n)=i$, where the first time we observe $\psi(n, \alpha) \downarrow$ is $\alpha=\omega \cdot i+j$ for some $j$. Let $q(i, n)$ be the first $m$ observed such that $\psi(n, \omega \cdot i+m) \downarrow$. The function $q$ is partial computable since it may be there is no such $m$ for the given $i$.

Let $\tilde{h}(i, x, n)$ denote the spot of $\emptyset^{b}$ which answers the question $\exists m \leq x[\psi(n$, $\omega \cdot i+m) \downarrow]$. Let $\tilde{r}(n, i)$ denote the spot of $\emptyset^{b}$ which answers the question $\exists m[\psi(n, \omega \cdot i+m) \downarrow]$. We then let $h(i, n)=\max \{\tilde{h}(i, x, n) \mid x \leq q(i, n)\}$ and $r(n)=\max \{\tilde{r}(n, x) \mid x \leq g(n)\}$. The functions $\tilde{h}, \tilde{r}$, and $r$ are computable, and $h$ is partial computable, converging wherever $q$ does.

Let $p(n)$ be the least $i$ such that for some $m$ we have $\psi(n, \omega \cdot i+m) \downarrow$. We can compute $p(n)$ from $\emptyset^{b} \| r(n)$. We note that $h(p(n), n)$ exists, and we can determine if $n \in A$ from $\emptyset^{b} \Uparrow \max \{r(n), h(p(n), n)\}$.

Let $v$ be a computable function defined by $\varphi_{v(i, n)}(y)=h(i, n)+r(n)(y$ is a dummy variable). Let $u(n)=\max \{v(i, n) \mid i \leq g(n)\}$. The function $u$ is computable, and if we let $j=v(p(n), n)$, then $j \leq u(n)$, the function $\varphi_{j}(y) \downarrow$, and $\emptyset^{b} \| \varphi_{j}(y)$ suffices to determine if $n \in A$ (for any $y$ ).

We now define $f(n)>u(n)$ to be such that (for any $y) \Phi_{f(n)}^{\emptyset^{b}}(y)$ runs the calculation to determine if $n \in A$ and converges iff $n \in A$. Explicitly, we define $f(n)>u(n)$ such that $\Phi_{f(n)}^{C}(y)$ is the partial computable function determined by the following steps. First, we let $x \leq g(n)$ be least such that $C(\tilde{r}(n, x))=1$. Next, we let $t$ be first such that we observe $\psi(n, \omega \cdot x+t) \downarrow$. We then let $z \leq t$ be least such that $C(\tilde{h}(x, z, n))=1$. Finally, we say that $\Phi_{f(n)}^{C}(y) \downarrow$ if $\psi(n, \omega \cdot x+z)=1$ 
and $\Phi_{f(n)}^{C}(y) \uparrow$ if $\psi(n, \omega \cdot x+z)=0$ (or if any of the above steps cannot be completed).

We note that $f$ is computable, and if $C$ is a sufficiently long initial segment of $\emptyset^{b}$, then $n \in A$ iff $\Phi_{f(n)}^{C}(y) \downarrow$. Recall that for any $n$, there exists $j \leq u(n)<f(n)$ such that $\varphi_{j}(y) \downarrow$, and $\emptyset^{b} \Uparrow \varphi_{j}(y)$ suffices to run the calculations to determine if $n \in A$.

We observe that

$$
\begin{aligned}
f(n) \in \emptyset^{2 b} & \Longleftrightarrow \quad \exists i \leq f(n)\left[\varphi_{i}(f(n)) \downarrow \wedge \Phi_{f(n)}^{\emptyset^{b} \Uparrow \varphi_{i}(f(n))}(f(n)) \downarrow\right] \\
& \Longleftrightarrow n \in A .
\end{aligned}
$$

Hence $f$ witnesses $A \leq{ }_{1} \emptyset^{2 b}$.

We use a similar method to prove the statement for all $k$.

Lemma 5.6 Let $k>1$, and let $A$ be a set such that $A$ is $\omega^{k}$-c.e. Then $A \leq_{1} \emptyset^{k b}$.

Proof We prove the statement by induction on $k$. The base case $(k=2)$ is given by Lemma 5.5. For the inductive case, we assume that the statement holds for $k$ and wish to show that it holds for $k+1$. We note for the procedure given in Lemma 5.5 that an index for $f$ can be computed uniformly from an index for $\psi$.

The proof for the inductive case proceeds along the same lines as the proof for the base case. Let $\psi$ witness that $A$ is $\omega^{k+1}$-c.e. Let $g$ be a computable function such that $g(n)=i$ where the first time we observe $\psi(n, \alpha) \downarrow$ is $\omega^{k} \cdot i+\alpha$ for some $\alpha<\omega^{k}$. Let $p(n)$ be the least $i$ such that for some $\alpha$ we have $\psi\left(n, \omega^{k} \cdot i+\alpha\right) \downarrow$.

Let $\chi_{i}(n, \alpha)=\psi\left(n, \omega^{k} \cdot i+\alpha\right)$ for all $\alpha<\omega^{k}$. We define a partial computable sequence of functions $e_{i}(n)$ as follows. To compute $e_{i}(n)$ we first search for any $\alpha$ such that $\chi_{i}(n, \alpha) \downarrow$. If there is none, then we must have $e_{i}(n) \uparrow$. If the search halts, then let

$$
\tilde{\chi}(m, \alpha)= \begin{cases}\chi_{i}(m, \alpha), & m=n, \\ 0 & \text { else. }\end{cases}
$$

Let $B$ be such that $\tilde{\chi}$ witnesses that $B$ is $\omega^{k}$-c.e., and let $\tilde{f}$ be given by applying the induction hypothesis to $B$. We then let $e_{i}(n)=\tilde{f}(n)$.

Let $v$ be a computable function defined by $\varphi_{v(i, n)}(y)=e_{i}(n)$ ( $y$ is a dummy variable). Let $u(n)=\max \{v(i, n) \mid i \leq g(n)\}$. The function $u$ is computable, and if we let $j=v(p(n), n)$, then $j \leq u(n)$, the function $\varphi_{j}(y) \downarrow$, and $\emptyset^{k b} \| \varphi_{j}(y)$ suffice to determine $e_{p(n)}(n)$ and hence if $n \in A$ (for any $y$ ).

We define $f(n)>u(n)$ to be such that (for any $y$ ), $\Phi_{f(n)}^{\emptyset^{k b}}(y)$ calculates if $n \in A$, and converges iff $n \in A$. Explicitly, we define $f(n)>u(n)$ such that $\Phi_{f(n)}^{C}(y)$ is the partial computable function determined by the following steps. Let $l$ be such that $\Phi_{l}^{\emptyset^{k b}}(m)=p(m)$. We then have $\Phi_{f(n)}^{C}(y)$ converge iff $\Phi_{l}^{C}(n)$ converges and $e_{\Phi_{l}^{C}(n)}(n) \in C$. As in the proof of Lemma 5.5, $f$ witnesses $A \leq_{1} \emptyset^{(k+1) b}$, completing the induction.

Therefore for all $k \geq 2$ we have that $A$ is $\omega^{k}$-c.e. $\Rightarrow A \leq_{1} \emptyset^{k b}$.

We proved earlier that for any set $A$ we had $A^{b_{0}} \leq_{1} A^{b}$ and $A^{b} \leq_{t t} A^{b_{0}}$. However, we can use the results above to show that $A^{b}$ and $A^{b_{0}}$ are not always 1-equivalent.

Remark 5.7 We have $\emptyset^{2 b} \not_{1}\left(\emptyset^{b}\right)^{b_{0}}$. 
Proof Suppose $\emptyset^{2 b} \leq_{1}\left(\emptyset^{b}\right)^{b_{0}}$. Let $A$ be a properly $\omega^{2}$-c.e. set. By Theorem 5.1, we have $A \leq{ }_{1} \emptyset^{2 b}$, so $A \leq_{1}\left(\emptyset^{b}\right)^{b_{0}}$.

Using an argument similar to the proof of Lemma 5.4, we can show that $\left(\emptyset^{b}\right)^{b_{0}}$ is $(\omega+1)$-c.e. Indeed, while $\varphi_{i}(j) \uparrow$ we believe $\langle e, i, j\rangle \notin\left(\emptyset^{b}\right)^{b_{0}}$, and once $\varphi_{i}(j) \downarrow$, since $\emptyset^{b}$ is c.e., we can approximate $\left(\emptyset^{b}\right)^{b_{0}}(\langle e, i, j\rangle)$ with at most $2\left(\varphi_{i}(j)\right)$-many changes. It is not hard to see that for any sets $B$ and $C$ and any ordinal $\alpha$, if $B \leq{ }_{1} C$ and $C$ is $\alpha$-c.e., then $B$ is $\alpha$-c.e. Hence $A$ is $(\omega+1)$-c.e., contradicting $A$ being properly $\omega^{2}$-c.e. We conclude that $\emptyset^{2 b} \not_{1}\left(\emptyset^{b}\right)^{b_{0}}$.

\section{Inversions}

We examine what type of inverses exist for the bounded jump. Anderson [1] proved that strong jump inversion holds for the truth-table degrees. For any set $X \geq_{t t} \emptyset^{\prime}$ there is a set $Y$ such that $X \equiv_{t t} Y^{\prime} \equiv_{t t} Y \oplus \emptyset^{\prime}$. It follows as a corollary that strong bounded jump inversion holds for the truth-table degrees.

Corollary 6.1 Let $X \geq_{t t} \emptyset^{b}$. Then there exists $Y$ such that $Y^{b} \equiv_{t t} X \equiv_{t t} Y \oplus$ $\emptyset^{b}$.

Proof Let $X$ be given, and let $Y$ be given by strong jump inversion for the truth-table degrees. Then $Y^{\prime} \equiv_{t t} X \equiv_{t t} Y \oplus \emptyset^{b}$, and from Section 4 we have $Y \oplus \emptyset^{b} \leq_{t t} Y^{b}$ and $Y^{b} \leq_{t t} Y^{\prime}$. We conclude that $Y^{b} \equiv_{t t} X \equiv_{t t} Y \oplus \emptyset^{b}$.

A close examination of the proof in [1] reveals that an equivalent statement also holds for the bounded Turing degrees. For any set $X \geq_{b T} \emptyset^{\prime}$ there is a set $Y$ such that $X \equiv_{b T} Y^{\prime} \equiv_{b T} Y \oplus \emptyset^{\prime}$. If we apply the proof of Corollary 6.1, we get that for any set $X \geq_{b T} \emptyset^{b}$ there is a set $Y$ such that $X \equiv_{b T} Y^{b} \equiv_{b T} Y \oplus \emptyset^{b}$.

As noted earlier, Shoenfield jump inversion (see [14]) holds for the Turing degrees with the Turing jump; for every $\Sigma_{2}$-set $X \geq_{T} \emptyset^{\prime}$ there is a $Y \leq_{T} \emptyset^{\prime}$ such that $Y^{\prime} \equiv_{T} X$. Csima, Downey, and $\mathrm{Ng}$ [5] showed that it does not hold for the bounded Turing degrees with the Turing jump.

We prove that Shoenfield jump inversion holds for the bounded Turing degrees with the bounded jump. In this example, the behavior of the bounded jump on the bounded Turing degrees more closely resembles the behavior of the Turing jump on the Turing degrees.

Theorem 6.2 Let $B$ be such that $\emptyset^{b} \leq_{b T} B \leq_{b T} \emptyset^{2 b}$. Then there is a set $A \leq_{b T} \emptyset^{b}$ such that $A^{b} \equiv_{b T} B$.

Proof Suppose $\emptyset^{b} \leq_{b T} B \leq_{b T} \emptyset^{2 b}$. Let $\psi$ witness that $B$ is $\omega^{2}$-c.e. We build an $\omega$-c.e. set $A$ (so $A \leq_{b T} \emptyset^{b}$ ) such that $A^{b} \equiv_{b T} B$.

We will define $A$ using a stage by stage construction. We will ensure that $A$ is $\omega$-c.e. via the function $f(x)=x+1$. Before we start, we define a computable function $g$. We will have $g$ witness that $B \leq_{1} A^{b}$.

For each $n \in \omega$, let $i_{n}$ be the first $i$ that we find such that $\psi(n, \omega \cdot i+j) \downarrow$, for some $j$. The definition of $\psi$ guarantees such an $i$ exists, so the $i_{n}$ are uniformly computable. We define an approximation $B_{s}$ for $B$ similarly. Fix $n$, and let $t$ be the least such that $\psi_{t}(n, \alpha) \downarrow$, for some $\alpha$. Given $s$, let $\tilde{s}=\max (t, s)$, and let $B_{s}(n)=\psi(n, \beta)$, where $\beta$ is the least such that $\psi_{\widetilde{s}}(n, \beta) \downarrow$.

We define a computable function $h$ to help define $g$. Let $g(-1)=-1$. Let $h(n)=\sum_{k=0}^{n-1} h(k)+\sum_{k=1}^{g(n-1)}\left(\frac{k^{2}-k}{2}\right)+i_{n}$. Let $g(n)$ be such that between $g(n-1)$ 
and $g(n)$ there are $h(n)$-many partial computable functions $\varphi_{k(n, 0)}, \ldots, \varphi_{k(n, h(n)-1)}$ that we control by the recursion theorem, and such that we control $\Phi_{g(n)}$ by the recursion theorem. The formal definitions of $g, h$, and $k$ are given at the end of the proof of Lemma 6.7.

We will make use of markers labeled $x_{n}^{i}$ with $i \leq i_{n}$, called $n$-markers, which will move stage by stage but reach a limit. At some stage $s$, we might say that a marker $x_{n}^{i}$ becomes defined. The marker then maintains its value, unless it becomes undefined at a later stage. If it at an even later stage becomes redefined, then it will have a new, larger value. At any moment, there will be at most one $n$-marker defined for each $n$. There will be a computable bound on the total number of times all $n$-markers will be defined/redefined, namely, $h(n)$.

In each stage of the construction we will make numerous changes to the approximation of the set $A$. To ease notation, when we write " $A$ " in the construction, we actually mean the most current approximation of $A$ at that moment of the construction. By " $A_{s}$ " we mean the approximation $A$ at the end of stage $s$. Without loss of generality, we assume that if $\psi_{s}(n, \omega \cdot i+j) \downarrow$, then the stage $s>j+1$.

Stage $s$

Step 1 . If some $\varphi_{e, s}(x) \downarrow$ for the first time at stage $s$, with $e \leq x \leq g(k)$, then for all $m>k$, extract all $x_{m}^{l}$ from $A$, and declare them undefined.

Step 2. Let $n \leq s$ be the least such that $x_{n}^{i}$ is defined but $A\left(x_{n}^{i}\right) \neq B_{s}(n)$, or such that $\psi(n, \alpha) \downarrow$ for some $\alpha$ but there is no marker defined for $n$. Let $\omega \cdot i+j$ be the least such that $\psi_{s}(n, \omega \cdot i+j) \downarrow$.

(a) If $x_{n}^{i}$ is undefined, then we perform the following steps. Define $x_{n}^{i}=s$. Extract all $x_{m}^{l}$ with $m>n$ and all $x_{n}^{k}$ with $k>i$ from $A$, and declare them undefined. Define $\varphi_{k(n, r)}(g(n)) \downarrow=x_{n}^{i}$ for some $r$, and declare $\Phi_{g(n)}^{\sigma^{\wedge}} 1(g(n)) \downarrow$ for every string $\sigma$ of length $x_{n}^{i}-1$. Note that by our assumption, $j+1<s=x_{n}^{i}$. There will always be some $r$ with $\varphi_{k(n, r)}(g(n))[s-1] \uparrow$ by our careful counting of $h(n)$.

(b) If needed, change $A_{s}\left(x_{n}^{i}\right)$ to ensure $x_{n}^{i} \in A_{s}$ iff $n \in B_{s}$ (so that $g(n) \in A_{s}^{b}$ iff $\left.n \in B_{S}\right)$.

This completes the construction.

Lemma 6.3 A is $\omega$-c.e.

Proof If at stage $s$ we did not set $s=x_{n}^{i}$ for any $n$, then $s$ was never enumerated into $A$. If at stage $s$ we set $s=x_{n}^{i}$, then $x_{n}^{i}$ is enumerated into $A$ and can be removed/enumerated into $A$ at most $j$-many more times by step 1 of the construction (where $j$ is the least such that $\psi_{s}(n, \omega \cdot i+j) \downarrow$ ). By convention $j \leq s$, so certainly $s$ is enumerated/removed from $A$ at most $(s+1)$-many times.

Lemma 6.4 For each $n$ and each $i \leq i_{n}, x_{n}^{i}=\lim x_{n}^{i}[s]$ exists, where we allow "undefined" as a possibility. Moreover, for each $n$, if $\tilde{\imath}_{n}=\mu i(\exists j)[\psi(n, \omega \cdot i+j) \downarrow]$ then $x_{n}^{i}$ is defined iff $i=\tilde{\imath}_{n}$, and $x_{n}^{\tilde{i}_{n}} \in A \Longleftrightarrow n \in B$. Finally, for each $n$, the total number of times any $n$-marker is defined or redefined, summing over all $i \leq i_{n}$, is at most $h(n)$.

Proof An $n$-marker $x_{n}^{i}$ can only become defined (redefined) via step 2(a) of the construction. Thus at the stage when $x_{n}^{i}$ is defined (redefined), $i$ is the least such that $\psi_{s}(n, \omega \cdot i+j) \downarrow$. At the moment that $x_{n}^{i}$ is defined (redefined), any $x_{n}^{k}$ with $k>i$ 
that may have been defined is undefined, and since $k>i$, will never be redefined at a later stage. That is, at any stage of the construction, there is at most one $i$ with $x_{n}^{i}$ defined, and, as a function of the stages, the index $i$ of the $n$-markers that are defined is nonincreasing. Since there is only one defined $n$-marker at any given stage, the total number of times that an $n$-marker is undefined by step 1 of the construction is bounded by $\sum_{k=1}^{g(n-1)}\left(\frac{k^{2}-k}{2}\right)$. Let $\hat{h}(m)$ be the total number of stages where an $m$ marker is defined (redefined). A 0-marker cannot be undefined by step 1 . In step 2, a 0 -marker can only be undefined if a new 0 -marker, with lower index, is defined. Thus $\hat{h}(0)=i_{0}=h(0)$. Similarly, $\hat{h}(n)=\sum_{k=1}^{g(n-1)}\left(\frac{k^{2}-k}{2}\right)+i_{n}+\sum_{k=0}^{n} h(k)=h(n)$.

Finally, consider $x_{n}^{\tilde{\tau}_{n}}$. Let $s$ be a stage by which all $m$-markers with $m \leq n$ have reached their limits, and such that $A_{t}\left\|x_{n}^{\widetilde{l}_{n}}=A_{s}\right\| x_{n}^{\widetilde{l}_{n}}$ for all $t \geq s$. Note that by definition of $\tilde{l}_{n}$, we have that $x_{n}^{\tilde{l}_{n}}$ is defined at stage $s$. Then by step 2(b) of the construction we have that $x_{n}^{\tilde{l}_{n}} \in A$ iff $n \in B$.

Lemma 6.5 We have $B \leq_{1} A^{b}$.

Proof We claim that $n \in B$ iff $g(n) \in A^{b}$. Consider the stage $s$ when $x_{n}^{\tilde{l}_{n}}$ was defined for the last time. At this stage, we set $\varphi_{k(n, r)}(g(n)) \downarrow=x_{n}^{\tau_{n}}$ for some $r$, and declare $\Phi_{g(n)}^{\sigma^{\wedge} 1}(g(n)) \downarrow$ for all $\sigma$ of length $x_{n}^{\tilde{\imath}}-1$. Since $k(n, r)<x_{n}^{\tilde{\imath}_{n}}$, we have that if $x_{n}^{\tilde{i}_{n}} \in A$, then $g(n) \in A^{b}$. Conversely, we only ever define $\Phi_{g(n)}(g(n))$ to halt in step 2(a) of the construction, and with an oracle that includes an $n$-marker. Since all $n$-markers besides $x_{n}^{\tilde{l}_{n}}$ were extracted from $A$ at stage $s$, we have that if $x_{n}^{\tilde{l}_{n}} \notin A$, then $g(n) \notin A^{b}$. Now by Lemma 6.4 we have $x_{n}^{\tilde{\imath}_{n}} \in A$ iff $n \in B$, so that $n \in B$ iff $g(n) \in A^{b}$, as desired.

Lemma 6.6 We have $A^{b} \leq_{b T} B$.

Proof Recall that $x \in A^{b} \Longleftrightarrow \exists e \leq x\left[\Phi_{x}^{A \Uparrow \varphi_{e}(x)}(x) \downarrow\right]$. Recall also that $\emptyset^{b} \leq_{b T} B$. Let $n$ be the least such that $x<g(n)$.

Let $k$ be the total number of different oracles that appear to witness $x \in A^{b}$ during the approximation of $A$. That is, $k$ is maximal such that

$$
\begin{aligned}
& \exists s_{1} \cdots \exists s_{k} \exists \sigma_{1} \cdots \exists \sigma_{k}\left(\sigma_{i} \neq \sigma_{j} \wedge \exists e \leq x\left[\varphi_{e, s_{i}}(x) \downarrow \wedge \sigma_{i}=A_{s_{i}} \Uparrow \varphi_{e, s_{i}}(x)\right]\right. \\
& \left.\quad \wedge \Phi_{x, s_{i}}^{\sigma_{i}}(x) \downarrow\right) .
\end{aligned}
$$

According to step 1 of the construction, whenever some $\varphi_{e}(x) \downarrow$ with $e \leq x$, all $m$-markers with $m>g(n)$ are extracted from $A$. So, if $x \in A^{b}$, then the only nonzero entries in the part of the oracle $A$ that is used in the computation are those that arise from $m$-markers with $m \leq n$. Since the total number of times $m$-markers can be redefined is bounded by $h(m)$, and since each marker can either be in or out of $A$, the number $k$ of possible oracles is computably bounded. (It is certainly bounded by $2^{\sum_{l=0}^{n} h(l)}$.) That is, we can $b T$-compute $k$ from $\emptyset^{b}$ and hence $B$ using questions of the form (1).

For each $m \leq n$, using at most $i_{m}$-many questions of the form

$$
\left(\exists x_{1}\right) \cdots\left(\exists x_{l}\right)\left[x_{p+1}<x_{p} \wedge(\exists j) \psi\left(m, \omega \cdot x_{p}+j\right) \downarrow\right],
$$

we can $b T$-compute $\tilde{l}_{m}$ from $\emptyset^{b}$ and hence $B$. 
Similarly, we can $b T$-compute from $\emptyset^{b}$, and hence from $B$, the number of pairs $e \leq y \leq g(n)$ such that $\varphi_{e}(y) \downarrow$. Thus we can $b T$-compute from $B$ the stage $s$, by which point if $e \leq y \leq g(n)$ and $\varphi_{e}(y) \downarrow$, then $\varphi_{e, s}(y) \downarrow$.

We can certainly $b T$-compute from $B$ the initial segment $B \Uparrow n$.

We now put the above facts together to compute whether $x \in A^{b}$. If $k=0$, then there is never any stage where it appears that $x \in A^{b}$, so $x \notin A^{b}$. So suppose $k \neq 0$. Run the approximation of $A$ to find the $k$-many different possible oracles which might witness $x \in A^{b}$. We know that the only possible nonzero entries in the correct oracle come from $x_{m}^{\tilde{\imath}_{m}}$ with $m \leq n$, and that $x_{m}^{\tilde{\imath}_{m}} \in A$ iff $m \in B$. Now since we have $b T$-computed from $B$ all the $\tilde{\imath}_{m}$ for $m \leq n$, we can run the approximation of $A$ until the least stage $t$ greater than $s$ where markers of the form $x_{m}^{\tau_{m}}$ are defined for all $m \leq n$. The location of $x_{m}^{\tau_{m}}$ at stage $t$ is its final location. Now, using $B \Uparrow n$, we have computed the true initial segment of $A$ that is relevant for deciding whether $x \in A^{b}$. If this oracle extends any of the $k$-many halting oracles that we found, then $x \in A^{b}$. Otherwise, $x \notin A^{b}$.

\section{Lemma 6.7 The functions $g, h$, and $k$ used in the construction exist.}

Proof Let $\Psi_{m, n, q}$ and $\Gamma_{n, q}$ denote the operations that are referred to in the main construction as $\varphi_{k(m, n)}$ and $\Phi_{g(n)}$, respectively, when the role of $g(n)$ in the construction (when not in the form $\Phi_{g(n)}$ ) is played by $\varphi_{q}(n)$. We wish to find $g, h$, and $k$ which satisfy the less than and greater than constraints in the main proof, and a number $i$ such that $\varphi_{k(m, n)}=\Psi_{m, n, i}, \Phi_{g(n)}=\Gamma_{n, i}$, and $g(n)=\varphi_{i}(n)$ for all $m, n$.

By the padding lemma, for each $n, m, q$ let $K_{n, m, q}$ be an infinite, uniformly computable set such that for all $l \in K_{n, m, q}$ we have $\varphi_{l}=\Psi_{n, m, q}$. Similarly, for all $n, q$ let $G_{n, q}$ be an infinite, uniformly computable set such that for all $l \in G_{n, q}$ we have $\Phi_{l}=\Gamma_{n, q}$.

We now define a uniformly computable procedure (in a parameter $q$ ) which we will label $\Theta_{q}$. The procedure will use simultaneous induction to define three computable functions, $\tilde{g}, \tilde{h}$, and $\tilde{k}$.

We start the procedure by saying $\tilde{g}(-1)=-1$. Given $\tilde{g}$ and $\tilde{h}$ up to $n-1$, we define $\tilde{h}(n)$ as we did in the main theorem, $\tilde{h}(n)=\sum_{t=0}^{n-1} \tilde{h}(t)+\sum_{t=1}^{\tilde{g}(n-1)}\left(\frac{t^{2}-t}{2}\right)+i_{n}$. Next, for each $m$ such that $0 \leq m<\tilde{h}(n)$ we assign the least possible element of $K_{n, m, q}$ as the value of $\tilde{k}(n, m)$ such that we satisfy $\tilde{g}(n-1)<\tilde{k}(n, 0)<\tilde{k}(n, 1)<$ $\cdots<\tilde{k}(n, \tilde{h}(n)-1)$. Finally we assign the least element of $G_{n, q}$ bigger than $\tilde{k}(n, \tilde{h}(n)-1)$ as the value of $\tilde{g}(n)$. This completes our induction and the procedure $\Theta_{q}$.

We note that if $\tilde{g}, \tilde{h}$, and $\tilde{k}$ come from procedure $\Theta_{q}$, then they meet the less than and greater than constraints in the main proof, and for all $m, n$ we have $\varphi_{\tilde{k}(n, m)}=\Psi_{n, m, q}$ and $\Phi_{\tilde{g}(n)}=\Gamma_{n, q}$.

Define a computable, injective function $w$ by letting $\varphi_{w(q)}(x)=\tilde{g}(x)$, where $\tilde{g}$ comes from procedure $\Theta_{q}$. Let $i$ be given by the recursion theorem applied to $w$. Finally, let $g, h$, and $k$ be given by $\tilde{g}, \tilde{h}$, and $\tilde{k}$ from procedure $\Theta_{i}$. Then $\varphi_{k(m, n)}=\Psi_{m, n, i}, \Phi_{g(n)}=\Gamma_{n, i}$, and $\varphi_{i}=\varphi_{w(i)}=g$, as desired.

We note that the proof above cannot be modified to find an $A$ such that $A^{b} \leq_{t t} B$. 


\section{Other Jump Operators}

In 1979, Gerla [7] proposed jump operators for the truth-table and bounded truthtable degrees. We wish to compare his observations on these operators with some of the results shown so far for the bounded jump. Since the original article is available only in Italian, we briefly summarize the definitions and highlight a few of the results from the paper.

We start with some basic definitions used in studying the truth-table degrees (see Rogers [12]).

Definition 7 A $t t$-condition is a finite sequence $x_{1} \cdots x_{k} \in \omega$ and a function $\alpha: 2^{k} \rightarrow 2$. We say that it is satisfied by $A$ if $\alpha\left(A\left(x_{1}\right) \cdots A\left(x_{k}\right)\right)=1$. We define $A^{t t}=\{x \mid x$ is a $t t$-condition satisfied by $A\}$.

We note that $A^{t t} \leq_{t t} A$ and $A \leq_{1} A^{t t}$. Gerla [7] uses $A^{t t}$ to define jumps $A_{t t}$ and $A_{b k}$ for the truth-table degrees and bounded truth-table degrees of norm $k$, respectively.

Definition 8 We have $A_{t t}=\left\{x \mid \varphi_{x}(x) \downarrow \in A^{t t}\right\}$ and $A_{b k}=\left\{x \mid \varphi_{x}(x) \downarrow \in\right.$ $\left.A^{t t} \wedge \varphi_{x}(x) \leq k\right\}$.

The behavior of $A_{t t}$ and $A_{b k}$ on the truth-table and bounded truth-table degrees shares several similarities with that of $A^{\prime}$ on the Turing degrees. We state a few of the many results below.

Theorem 7.1 (Gerla [7]) Let $k$ be a number, and let $A$ and $B$ be sets:
1. $A_{t t} \underline{L}_{t t} A, A_{b k} \varliminf_{b k} A$;
2. $A \leq_{t t} B \Rightarrow A_{t t} \leq_{1} B_{t t}$;
3. $A<_{1} A_{b k} \leq_{1} A_{b(k+1)} \leq_{1} A_{t t} \leq_{1} A^{\prime}$;
4. $\emptyset_{b k} \equiv_{1} \emptyset_{t t} \equiv_{1} \emptyset^{\prime}$.

We demonstrated earlier the connection between the bounded jump and the Ershov hierarchy. We see that the finite levels of the Ershov hierarchy share a similar (but weaker) connection with $A_{b k}$.

Theorem 7.2 (Gerla [7]) Let $A$ be $n$-c.e., and let $B \leq_{1} A_{b k}$. Then $B$ is $(n k+1)$ c.e.

Let $\emptyset_{n(b k)}$ denote the $n$th iteration of the $b k$-jump of the empty set. It follows from the theorem that if $A \leq_{1} \emptyset_{n(b k)}$, then $A$ is $\left(1+k+k^{2}+\cdots+k^{n-1}\right)$-c.e. (see [7]).

Since Gerla's truth-table jump is designed for a stronger reducibility, we expect it to be weaker than the bounded jump. We prove that for every set $A$ we have $A_{t t} \leq_{1} A^{b}$, but there are many sets $X$ such that $X^{b} \sum_{b T} X_{t t}$.

Proposition 7.3 We have $A_{t t} \leq_{1} A^{b_{0}}$.

Proof Let $f$ and $\Phi_{k}$ witness that $A^{t t} \leq_{b T} A$. We define computable, injective functions $h$ and $j$. Let $\varphi_{h(e)}(z)=f\left(\varphi_{e}(e)\right)$.

Define $j$ by $\Phi_{j(e)}^{C}(z) \downarrow$ iff $\varphi_{e}(e) \downarrow$ and $\varphi_{e}(e) \in \Phi_{k}^{C}$.

Let $z$ represent an arbitrary dummy variable. We note the following:

$$
\begin{aligned}
x \in A_{t t} & \Longleftrightarrow \varphi_{x}(x) \downarrow \in A^{t t} \\
& \Longleftrightarrow \varphi_{x}(x) \downarrow \in \Phi_{k}^{A}
\end{aligned}
$$




$$
\begin{array}{ll} 
& \Longleftrightarrow \quad \varphi_{x}(x) \downarrow \in \Phi_{k}^{A \Uparrow f\left(\varphi_{x}(x)\right)} \\
& \Longleftrightarrow \quad \varphi_{x}(x) \downarrow \in \Phi_{k}^{A \Uparrow \varphi_{h(x)}(z)} \\
& \Longleftrightarrow \quad \Phi_{j(x)}^{A \Uparrow \varphi_{h(x)}(z)}(z) \downarrow \\
& \Longleftrightarrow \quad\langle j(x), h(x), z\rangle \in A^{b_{0}} .
\end{array}
$$

Thus $A_{t t} \leq_{1} A^{b_{0}}$.

Corollary 7.4 We have $A_{t t} \leq_{1} A^{b}$.

Theorem 7.5 There is a c.e. set $A$ such that $A^{b} \bigsqcup_{b T} A_{t t}$.

Proof First note that if we have a computable approximation to a set $A$, then this induces an obvious approximation for $A_{t t}$. Namely, if $\varphi_{x, s}(x) \uparrow$, then $x \notin A_{t t}[s]$, and if $\varphi_{x, s}(x) \downarrow$, then $x \in A_{t t}[s] \Longleftrightarrow \varphi_{x}(x) \in A[s]^{t t}$. We also have an approximation for $A^{b}$ by $x \in A^{b}[s] \Longleftrightarrow \exists i \leq x\left[\varphi_{i, s}(x) \downarrow \wedge \Phi_{x}^{A \Uparrow \varphi_{i, s}(x)}(x) \downarrow[s]\right]$. We note that if $A$ is c.e., then these are both $\Delta_{2}^{0}$-approximations.

For $k \in \omega$, let $l(k, s)=\max \left\{\varphi_{x, s}(x) \mid x \leq k \wedge \varphi_{x, s}(x) \downarrow\right\}$. Note that for $s<t$, if $A \| l(k, s)[s]=A \Uparrow l(k, s)[t]$, then $A_{t t}\left\|k[s]=A_{t t}\right\| k[t]$ unless $\varphi_{x, t}(x) \downarrow$ for some $x \leq k$ such that $\varphi_{x, s}(x) \uparrow$.

We now proceed with the construction of $A$. We must meet for all $n \in \omega$ the requirement

$$
R_{n}:(\neg \forall x)\left[\varphi_{\pi_{2}(n)}(x) \downarrow \wedge \Phi_{\pi_{1}(n)}^{A_{t t} \| \varphi_{\pi_{2}(n)}(x)}(x)=A^{b}(x)\right],
$$

where $\pi_{1}$ and $\pi_{2}$ are projection functions for some canonical pairing function.

To ease notation, we will use the following convention. We write $\Phi_{i}$ for $\Phi_{\pi_{1}(i)}$. For $\varphi$ we distinguish between two cases. We write $\varphi_{i}\left(x_{i}\right)$ for $\varphi_{\pi_{2}(i)}\left(x_{i}\right)$. However, $\varphi_{y}(y)$ maintains its usual meaning for any $y$.

Let $e_{0}<e_{1}<e_{2}<\cdots$ be a computable list such that we control $\varphi_{e_{i}}$ and $\Phi_{e_{i}}$ by the recursion theorem. A formal definition can be accomplished by the methods used in Lemma 6.7.

We will use a set of movable markers $x_{i}$ for $i \in \omega$ such that for all $i$ we have $x_{i}=e_{j}$ for some $j$. We will also make use of a restraint function $r$. We start with $r(n)[0]=0$ for all $n$.

Stage 0. Let $x_{0}=e_{0}$.

Stage $s+1$. For each $m$ let $r(m)[s+1]=\max \left\{\varphi_{x, s+1}(x) \mid x \leq \varphi_{l}\left(x_{l}\right)[s]\right.$ for some $l<m\}$. (We say that $r(m)[s+1]=0$ if this set is empty.) Let $k$ be the least such that $r(k)[s+1]>r(k)[s]$. (If no such $k$ exists, use $k=s$.) Undefine all $x_{m}$ with $m \geq k$.

Case 1. There is no $n<k$ such that $x_{n}$ is defined and $\Phi_{n}^{A_{t t} \Uparrow \varphi_{n}\left(x_{n}\right)}\left(x_{n}\right)[s] \downarrow=$ $A^{b}\left(x_{n}\right)[s]$.

We then let $m$ be the least such that $x_{m}$ is not defined, and define $x_{m}$ to be the least $e_{i}$ that has not been used in the construction. (Proceed to the next stage.)

Case 2. Else.

We then let $n<k$ be least such that $x_{n}$ is defined and $\Phi_{n}^{A_{t t} \| \varphi_{n}\left(x_{n}\right)}\left(x_{n}\right)[s] \downarrow=$ $A^{b}\left(x_{n}\right)[s]$. Undefine all $x_{m}$ with $m>n$. If it has not yet been defined (with the current value of $\left.x_{n}\right)$, let $\varphi_{x_{n}}\left(x_{n}\right)=r(n)[s+1]+\max \left\{A_{s}\right\}+\varphi_{n}\left(x_{n}\right)$.

Subcase 2A. We have $A^{b}\left(x_{n}\right)[s]=0$.

Set $\Phi_{x_{n}}^{A_{s} \| \varphi_{x_{n}}\left(x_{n}\right)}\left(x_{n}\right) \downarrow$, so that $A^{b}\left(x_{n}\right)[s+1]=1$. 
Subcase 2 B. $A^{b}\left(x_{n}\right)[s]=1$.

Choose the least $x>r(n)$ such that $x \notin A[s]$, and enumerate $x \in A[s+1]$. We demonstrate later that we have $x \leq \varphi_{x_{n}}\left(x_{n}\right)$, so that this will cause $A^{b}\left(x_{n}\right)[s+1]=0$.

This completes the construction of $A$.

It is easy to see that the set constructed is c.e. We claim that for each $n$, $x_{n}=\lim _{s} x_{n}[s]$ exists and provides a witness for $R_{n}$. We say that a requirement $R_{n}$ receives attention if we perform case 2 of the construction for some $x_{n}$.

Lemma 7.6 For each $n, x_{n}=\lim _{s} x_{n}[s]$ exists and provides a witness for $R_{n}$. Moreover, the requirement $R_{n}$ receives attention at most finitely often.

Proof Since $x_{0}$ is never undefined, it reaches its limit at stage 0. Assume that $x_{l}$ with $l<m$ have reached their limit, and if $\varphi_{l}\left(x_{l}\right) \downarrow$, then it has already done so. Then the value $r(m)$ can increase at $\operatorname{most} \max \left\{\varphi_{l}\left(x_{l}\right) \mid l<m\right\}$-many more times, and so there must be a stage after which $x_{m}$ is never undefined.

Assume for a contradiction that $x_{n}$ is the least such that $\Phi_{n}^{A_{t t} \| \varphi_{n}\left(x_{n}\right)}\left(x_{n}\right) \downarrow=$ $A^{b}\left(x_{n}\right)$. Let $s$ be the least stage after which no $x_{m}$ with $m<n$ requires attention and $r(n)$ has reached its limit. So at stage $s+1$ of the construction, $x_{n}$ is defined and is never again undefined. Since $\Phi_{n}^{A_{t t} \Uparrow \varphi_{n}\left(x_{n}\right)}\left(x_{n}\right) \downarrow=A^{b}\left(x_{n}\right)$, there is a least stage $s_{0}>s+1$ such that $\Phi_{n}^{A_{t t} \Uparrow \varphi_{n}\left(x_{n}\right)}\left(x_{n}\right)\left[s_{0}\right] \downarrow=A^{b}\left(x_{n}\right)\left[s_{0}\right]$. Since $s_{0}$ is the first stage where $R_{n}$ requires attention with this value of $x_{n}$, we define $\varphi_{x_{n}}\left(x_{n}\right)=r(n)+\max \left\{A_{s_{0}-1}\right\}+\varphi_{n}\left(x_{n}\right)$ at stage $s_{0}$, and we have $A^{b}\left(x_{n}\right)\left[s_{0}-1\right]=0$. So at stage $s_{0}$ of the construction, we set $\Phi_{x_{n}}^{A_{s_{0}} \| \varphi_{x_{n}}\left(x_{n}\right)}\left(x_{n}\right) \downarrow$, so that $A^{b}\left(x_{n}\right)\left[s_{0}\right]=1$. Note that at stage $s_{0}$ there are at least $\varphi_{n}\left(x_{n}\right)$-many numbers greater than $r(n)$ and less than $\varphi_{x_{n}}\left(x_{n}\right)$ available to enumerate into $A$.

Let $s_{0}<s_{1}<s_{2}<\cdots$ be all the further stages of the construction where $R_{n}$ receives attention. We will show that for all even $k$ an element is enumerated into $\emptyset^{\prime} \| \varphi_{n}\left(x_{n}\right)$ at some stage $t$ with $s_{k}<t \leq s_{k+1}$. It follows that the sequence $s_{0}, s_{1}, \ldots$ must be finite, contradicting the assumption that $\Phi_{n}^{A_{t t} \| \varphi_{n}\left(x_{n}\right)}\left(x_{n}\right) \downarrow=A^{b}\left(x_{n}\right)$. We will also show inductively that there is sufficient room to enumerate elements into $A$ between $r(n)$ and $\varphi_{x_{n}}\left(x_{n}\right)$, as claimed earlier.

Let $k$ be even, and assume for our induction that there are at least $\left(\varphi_{n}\left(x_{n}\right)-\frac{k}{2}\right)$ many numbers greater than $r(n)$ and less than $\varphi_{x_{n}}\left(x_{n}\right)$ available to enumerate into $A$. Without loss of generality, suppose that at stage $s_{k}$ we ensured $A^{b}\left(x_{n}\right)\left[s_{k}\right]=1$. Since all requirements $R_{m}$ with $m<n$ have stopped acting, no requirement $R_{m}$ with $m \leq n$ enumerated into $A$ at any stage $s_{k} \leq t \leq s_{k+1}$. Furthermore, since $r(m) \geq \varphi_{x_{n}}\left(x_{n}\right)$ for all $m>n$, no requirement $R_{m}$ enumerates into $A \Uparrow \varphi_{x_{n}}\left(x_{n}\right)$ at any stage $s_{k} \leq t<s_{k+1}$. Hence $A^{b}\left(x_{n}\right)\left[s_{k+1}-1\right]=1$ and $\Phi_{n}^{A_{t t} \| \varphi_{n}\left(x_{n}\right)}\left(x_{n}\right)\left[s_{k+1}-1\right]=1$. So $A_{t t}\left\|\varphi_{n}\left(x_{n}\right)\left[s_{k}-1\right] \neq A_{t t}\right\| \varphi_{n}\left(x_{n}\right)\left[s_{k+1}-1\right]$.

Using our observation from the start of the proof of the theorem, to demonstrate that there is a $y \leq \varphi_{n}\left(x_{n}\right)$ such that $\varphi_{y, s_{k}}(y) \uparrow$ but $\varphi_{y, s_{k+1}}(y) \downarrow$, it suffices to show $A \Uparrow l\left(\varphi_{n}\left(x_{n}\right), s_{k}-1\right)\left[s_{k}-1\right]=A \Uparrow l\left(\varphi_{n}\left(x_{n}\right), s_{k+1}-1\right)\left[s_{k+1}-1\right]$. Between stages $s_{k}-1$ and $s_{k+1}-1$, the construction only runs subcase 2 for a requirement $R_{m}$ with $m>n$. Hence no element is enumerated into $A \Uparrow r(n+1)$. Since $r(n+1) \geq l\left(\varphi_{n}\left(x_{n}\right), s_{k}-1\right)$ we have $A \Uparrow l\left(\varphi_{n}\left(x_{n}\right), s_{k}-1\right)\left[s_{k}-1\right]=A \Uparrow l\left(\varphi_{n}\left(x_{n}\right)\right.$, 
$\left.s_{k+1}-1\right)\left[s_{k+1}-1\right]$, as desired. Thus some $y \leq \varphi_{n}\left(x_{n}\right)$ was added to $\emptyset^{\prime}$ between stages $s_{k}$ and $s_{k+1}$.

At stage $s_{k+1}$ the least $x>r(n)$ such that $x \notin A\left[s_{k+1}-1\right]$ was enumerated into $A\left[s_{k+1}\right]$. By the induction hypothesis, we had $x \leq \varphi_{x_{n}}\left(x_{n}\right)$, so that $A^{b}\left(x_{n}\right)\left[s_{k+1}\right]=0$. Note that at stage $s_{k+1}+1$ there are at least $\left(\varphi_{n}\left(x_{n}\right)-\frac{k}{2}-1\right)$ many numbers greater than $r(n)$ and less than $\varphi_{x_{n}}\left(x_{n}\right)$ available to enumerate into $A$.

At stage $s_{k+2}$, we acted because $\Phi_{n}^{A_{t t} \|_{n}\left(x_{n}\right)}\left(x_{n}\right)\left[s_{k+2}-1\right] \downarrow=A^{b}\left(x_{n}\right)\left[s_{k+2}-\right.$ $1]=0$. We set $\Phi_{x_{n}}^{A_{s_{k+2}} \| \varphi_{x_{n}}\left(x_{n}\right)}\left(x_{n}\right) \downarrow$, so that $A^{b}\left(x_{n}\right)\left[s_{k+2}\right]=1$. There was no enumeration into $A$ below $r(n+1)$ at any stage $s_{k+1}<t \leq s_{k+2}$, so that at stage $s_{k+2}+1$ there are at least $\left(\varphi_{n}\left(x_{n}\right)-\frac{k}{2}-1\right)$-many numbers greater than $r(n)$ and less than $\varphi_{x_{n}}\left(x_{n}\right)$ available to enumerate into $A$. This completes our induction.

Since we can only reach a stage $s_{k}$ with $k$ even if a number less than $\varphi_{n}\left(x_{n}\right)$ enters $\emptyset^{\prime}$, and since we have left room to enumerate into $A$ in the desired interval at each such stage, it follows that there can be only finitely many stages $s_{k}$, as desired.

A similar proof can be used to show that every 2-generic $A$ is such that $A^{b} \varliminf_{b T} A_{t t}$.

Finally, we note that the minijump operator developed by Ershov [6] works on the $p m$-degrees in a manner similar to $A_{t t}$ on the truth-table degrees (see [11, Volume II, p. 732]).

\section{Further Study}

There is considerable room left to explore in the study of the bounded jump. We can examine to what degree do important results for the Turing jump on the Turing degrees also hold for the bounded jump on the bounded Turing degrees, particularly in cases where these results do not hold for the Turing jump on the bounded Turing degrees.

For example, Sacks [13] proved that for every $\Sigma_{2}$-set $X \geq_{T} \emptyset^{\prime}$ there is a c.e. set $Y$ such that $Y^{\prime} \equiv_{T} X$. Csima, Downey, and $\mathrm{Ng}$ [5] proved that Sacks jump inversion does not hold for the Turing jump on the bounded Turing degrees. We do not know if Theorem 6.2 holds if we add the requirement that $Y$ is c.e.

We can also look at concepts related to the Turing jump. We say that a set $X$ is bounded high if $X^{b} \geq_{b T} \emptyset^{2 b}$ and bounded low if $X^{b} \leq_{b T} \emptyset^{b}$. We can then attempt to characterize which sets are bounded high and bounded low. Finally, the jumps for the truth-table and bounded truth-table degrees developed by Gerla [7] could be considered in more detail.

\section{References}

[1] Anderson, B. A., "Automorphisms of the truth-table degrees are fixed on a cone," Journal of Symbolic Logic, vol. 74 (2009), pp. 679-88. MR 2518818. DOI 10.2178/js1/1243948334. 246, 256

[2] Ash, C. J., and J. Knight, Computable Structures and the Hyperarithmetical Hierarchy, vol. 144 of Studies in Logic and the Foundations of Mathematics, North-Holland, Amsterdam, 2000. MR 1767842. 252

[3] Coles, R., R. Downey, and G. Laforte, personal communication, May 2010. 
[4] Cooper, B., Computability Theory, Chapman and Hall/CRC, Boca Raton, Fla., 2004. MR 2017461. 247

[5] Csima, B., R. Downey, and K. M. Ng, "Limits on jump inversion for strong reducibilities,” Journal of Symbolic Logic, vol. 76 (2011), pp. 1287-96. MR 2895396. DOI 10.2178/js1/1318338849. 246, 256, 263

[6] Ershov, Y. L., "On a hierarchy of sets, III," Algebra and Logic, vol. 9 (1970), pp. 20-31. 263

[7] Gerla, G., "Una generalizzazione della gerarchia di Ershov," Bollettino della Unione Matematica Italiana (9), vol. 16-B (1979), pp. 765-78. 260, 263

[8] Jockusch, Jr., C. G., and R. A. Shore, "Pseudojump operators, II: Transfinite iterations, hierarchies, and minimal covers," Journal of Symbolic Logic, vol. 49 (1984), pp. 120536. MR 0771789. DOI 10.2307/2274273. 252

[9] Mohrherr, J., "Density of a final segment of the truth-table degrees," Pacific Journal of Mathematics, vol. 115 (1984), pp. 409-19. MR 0765197. 246

[10] Nies, A., Computability and Randomness, vol. 51 of Oxford Logic Guides, Oxford University Press, Oxford, 2009. MR 2548883.

DOI 10.1093/acprof:oso/9780199230761.001.0001. 250, 252

[11] Odifreddi, P. G., Classical Recursion Theory, Vol. II, vol. 143 of Studies in Logic and the Foundations of Mathematics, North-Holland, Amsterdam, 1999. 252, 263

[12] Rogers, Jr., H., Theory of Recursive Functions and Effective Computability, 2nd edition, MIT Press, Cambridge, Mass., 1987. MR 0224462. 260

[13] Sacks, G. E., "Recursive enumerability and the jump operator," Transactions of the American Mathematical Society, vol. 108 (1963), pp. 223-39. MR 0155747. 263

[14] Shoenfield, J. R., "On degrees of unsolvability", Annals of Mathematics (2), vol. 69 (1959), pp. 644-53. MR 0105355. 246, 256

[15] Soare, R. I., Recursively Enumerable Sets and Degrees: A Study of Computable Functions and Computably Generated Sets, Perspectives in Mathematical Logic, Springer, Berlin, 1987. MR 0882921. 246, 247

[16] Soare, R. I., Computability Theory and Applications, forthcoming, Springer-Verlag, preprint. 246, 247

\section{Acknowledgments}

B. Csima was partially supported by Natural Sciences and Engineering Research Council of Canada Discovery Grant 312501. B. Csima would like to thank the Max Planck Institute for Mathematics, Bonn, Germany, for a productive visit.

Anderson

Division of Mathematics and Natural Sciences

Gordon State College

419 College Drive

Barnesville, Georgia 30204

USA

banderson@gordonstate.edu

www.gordonstate.edu/Faculty/banderson

Csima

Department of Pure Mathematics

University of Waterloo

200 University Avenue W

Waterloo, Ontario N2L 3G1

Canada

csima@uwaterloo.ca

www. math.uwaterloo.ca/ csima 